\title{
Identification of adeno-associated virus contamination in cell and virus stocks by PCR
}

\author{
Hisako Katano, Sandra Afione, Michael Schmidt, and John A. Chiorini \\ National Institutes of Health, Bethesda, MD, USA
}

BioTechniques 36:676-680 (April 2004)

To further understand the biology of adeno-associated virus (AAV) and identify the presence of AAV in laboratory samples, we have developed a sensitive PCR-based assay using degenerate primers based on the sequence of seven diverse $A A V$ isolates. Using these primers, we can detect free virus in viral stocks, cleared cell lysate, as well as in latently infected cells. The method can detect as little as 10 viral copies/ $\mu L$ of sample and can be adapted for highthroughput screening technology. With this method, we have also detected a new AAV isolate from a stock of bovine adenovirus.

\section{INTRODUCTION}

Adeno-associated viruses (AAVs) are members of the Parvovirdae family and are helper virus-dependent for replication (for a review, see Reference 1). The genome consists of two open reading frames (ORFs) that encode the regulatory and structural proteins of the virus. The life cycle of AAVs is biphasic. In the absence of a helper virus, AAVs enter a quiescent latent state in the cell. However, upon the co-infection with a helper virus such as adenovirus (Ad) or herpes simplex virus (HSV), AAVs are reactivated and replicate to high copy number in the cell.

A hallmark of the life cycle of AAV2 is its interaction and inhibition of replication of other viruses. During a co-infection, AAV can inhibit both Ad and simian virus 40 (SV40) propagation (2-7), while expression of AAV replication (Rep) proteins is sufficient to inhibit the DNA replication of HSV (8), bovine papillomavirus (9), human papillomavirus (10), and human immunodeficiency virus $(11,12)$. Overexpression of the regulatory proteins of AAV2 (Rep78, 68, 52, and 40) can have a negative impact on cell growth and gene expression as well as the ability to transform cells using papillomavirus or by
Ad E1a plus an activated ras oncogene (13-15). Furthermore, the detection of AAV samples in clinical samples is important with regard to the use of AAV vectors in ongoing clinical trials. Therefore, the identification of AAV contamination in laboratory samples is of importance for studies involving viral and cell growth and metabolic activities.

Our goal in this study was to develop a sensitive PCR-based assay using degenerate primers based on the sequence of seven diverse AAV isolates. Using these primers, we can detect both free virus in viral stocks, cleared cell lysate, as well as in latently infected cells. The method can detect as little as 10 viral copies/ $\mu \mathrm{L}$ of sample and can be adapted for high-throughput screening technology. Furthermore, the primers have high specificity, and we have used them to detect AAV of an unknown serotype in a stock of bovine Ad.

\section{MATERIALS AND METHODS}

\section{Polymerase Chain Reaction}

For PCR of viral stocks, viral DNA

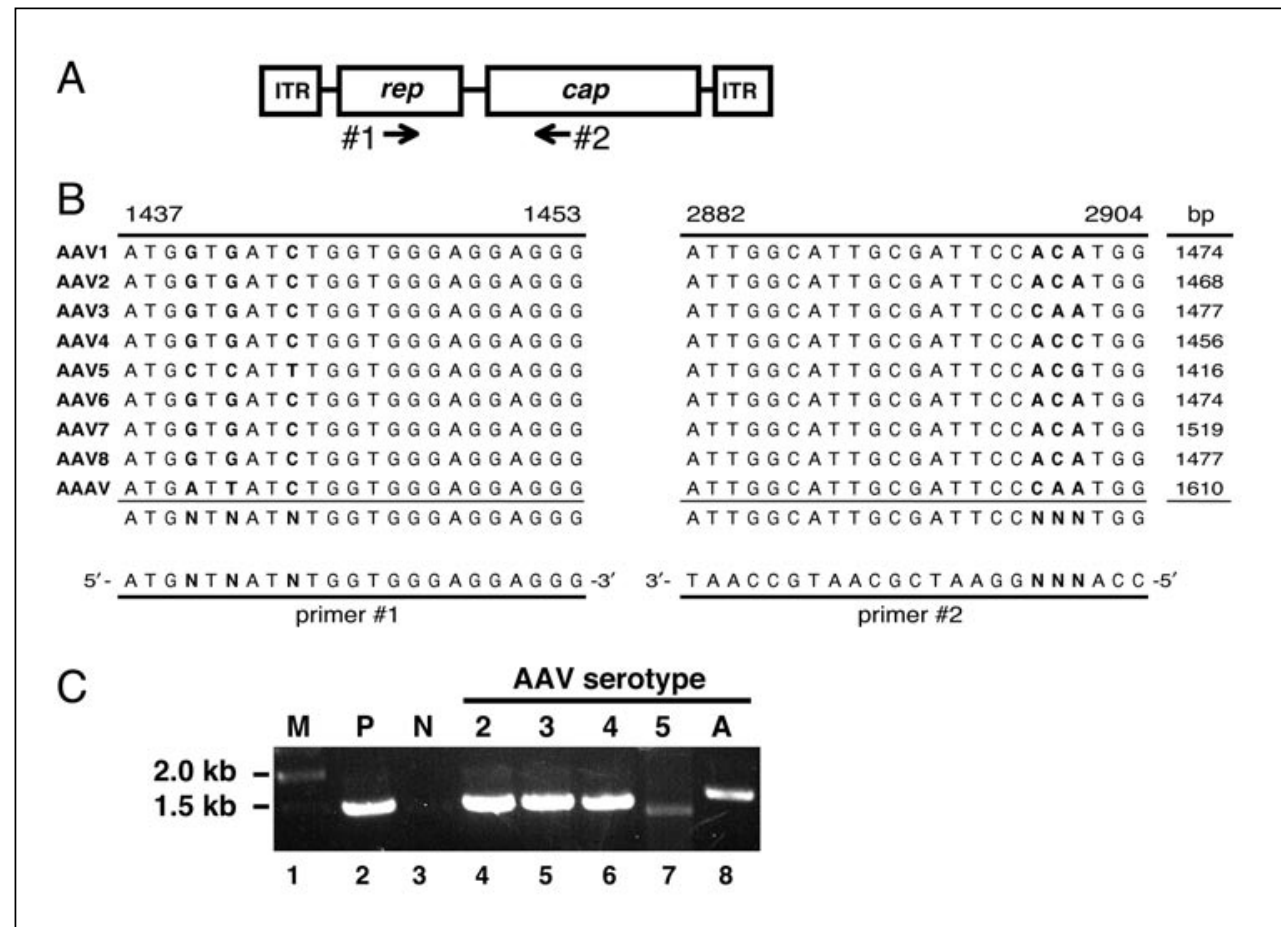

Figure 1. Summary of the PCR-based assay. (A) Universal primers. The adeno-associated virus (AAV) genomes contain two open reading frames (ORFs), rep and cap, flanked by inverted terminal repeats (ITRs). The locations of the AAV-specific primers, \#1 (forward) and \#2 (reverse), are indicated on the map by arrows. (B) Sequence of the AAV-specific primers. The genomes of AAV1-8 and avian AAV (AAAV) were aligned as described previously (23), with the AAV2 map position indicated at the top. A divergent nucleotide between the isolates is shown in bold capital letters and corresponds to an $\mathrm{N}$ in the universal primer below the alignments. The actual primers used in this study are shown in their orientations on the bottom. The size of PCR product expected to each serotype is shown on the right. (C) Detection of AAV DNA. Isolate-specific DNA was extracted from clarified cell lysate following the production of AAV. M, molecular size marker; P, purified wild-type AAV2; N, no template DNA control; 2, recombinant AAV2cleared viral lysate (rAAV2-CVL); 3, rAAV3-CVL; 4, rAAV4-CVL; 5, rAAV5-CVL; A, rAAAV-CVL. 
was first extracted from the sample using the QIAprep ${ }^{\circledR}$ Spin Miniprep kit (Qiagen, Valencia, CA, USA) with the following modification. The volume of P1 buffer was reduced to 150 $\mu \mathrm{L}$ to account for the $100-\mu \mathrm{L}$ volume of the cleared viral lysates (CVLs) (total volume $250 \mu \mathrm{L})$. The PCR $(50 \mu \mathrm{L})$ contained $1 \mu \mathrm{L}$ DNA template, 22.8 $\mu \mathrm{L}$ PCR-grade water, $10 \mu \mathrm{L} \mathrm{GC} \mathrm{Ge-}$ nomic PCR buffer (5×), $25 \mathrm{mM} 2.2 \mu \mathrm{L}$ $\mathrm{Mg}(\mathrm{OAc})_{2}, 5 \mathrm{M} 10 \mu \mathrm{L}$ GC-Melt, $2 \mu \mathrm{L}$ primer mixture [5'-ATGNTNATNTGGTGGGAGGAGGG-3' (forward) and 5'-CCANNNGGAATCGCAATGCCAAT-3' (reverse) $(25 \mu \mathrm{M}$ each)], 1 $\mu \mathrm{L}$ dNTP mixture $(10 \mathrm{mM}$ each), and $1 \mu \mathrm{L}$ Advantage-GCTM Genomic Polymerase Mixture (50×) (BD Biosciences Clontech, Palo Alto, CA, USA). The cycling conditions were $94^{\circ} \mathrm{C}$ for 5 min, followed by 10 cycles of $94^{\circ} \mathrm{C}$ for $30 \mathrm{~s}, 50^{\circ} \mathrm{C}$ for $30 \mathrm{~s}$, and $72^{\circ} \mathrm{C}$ for $2.5 \mathrm{~min}$, followed by 25 cycles of $94^{\circ} \mathrm{C}$ for $30 \mathrm{~s}, 54^{\circ} \mathrm{C}$ for $30 \mathrm{~s}$, and $72^{\circ} \mathrm{C}$ for $2.5 \mathrm{~min}$, terminating in $72^{\circ} \mathrm{C}$ for $5 \mathrm{~min}$. A robust PCR product was observed in DNA from latently infected cells following the addition of five extra cycles to the second round of PCR ( 30 cycles instead of 25).

\section{Production of Adenovirus-Free Wild-Type AAV}

Wild-type AAV2 was produced by a cotransfection of a full-length AAV2 plasmid (pAV2) and an Ad helper plasmid, purified by $\mathrm{CsCl}$ isopycnic centrifugation, and titered by quantitative PCR as previously described $(7,16)$. Briefly, semiconfluent 293T cells are transfected by calcium phosphate with three plasmids: (i) an $\mathrm{Ad}$ helper plasmid containing the virusassociated (VA) RNA, E2 and E4; (ii) an AAV helper plasmid containing the rep and cap genes for the serotype that is to be packaged; and (iii) a vector plasmid containing the inverted terminal repeats (ITRs) corresponding to the serotypes flanking a reporter gene of interest. Forty-eight hours posttransduction, the cells are harvested by scraping in TD buffer $(25 \mathrm{mM}$ Tris$\mathrm{HCl}, \mathrm{pH} 7.4,140 \mathrm{mM} \mathrm{NaCl}, 5 \mathrm{mM}$ $\mathrm{KCl}, 0.7 \mathrm{mM} \mathrm{K}_{2} \mathrm{HPO}_{4}$ ), and the cell pellet is concentrated by low-speed centrifugation.

\section{Generation of Clarified Cell or Viral Lysate}

The cell pellet $\left(3 \times 10^{8}\right.$ cells/plate $)$ was resuspended in TD buffer $\left(2 \times 10^{7}\right.$ cells $/ \mathrm{mL}$ ), and the cell lysate was frozen and thawed three times. Cellular debris was removed by centrifugation $(2000 \times g)$.

\section{Cells}

Human lung adenocarcinoma (EXVK) cells were maintained in RPMI 1640 supplemented with 5\% fetal calf serum (FCS). Latently infected cells were established by infecting cells with wild-type AAV2 [multiplicity of infection (MOI) 5000], and 48 $\mathrm{h}$ postinfection, individual cell clones were isolated by limiting dilution and maintained for 8 weeks (greater than three cell passages) prior to assaying for AAV DNA. Latently infected clones were identified by their ability to produce wild-type AAV particles upon super infection with Ad using PCR.

\section{RESULTS}

To date, eight AAV serotypes have been cloned and shown to have tropism for tissues as diverse as muscle, lung, liver, and brain (17-20). More recently, a large number of partial genome sequences were identified in nonhuman primates using a set of PCR primers based on reported sequence for AAVs 1-4 and AAV6, suggesting that AAV genomes maybe widespread (21).

To develop a sensitive and universal system for the detection of AAV contamination, the viral genomes of AAV1-8 and avian AAV (AAAV) were aligned using ClustalX (in PcGene; IntelliGenetics, Mountain View, CA, USA) (22) and scanned for regions of strong sequence conservation separated by a divergent region. A set of primers was selected that spans the end of the rep ORF through the beginning of the cap ORF (Figure1, A and B). To check for specificity, a computer pattern search, Basic Local Alignment Search Tool (BLAST; National Center for Biotechnology Information, National Library of Medicine, National Institutes of Health), with the primers did not 


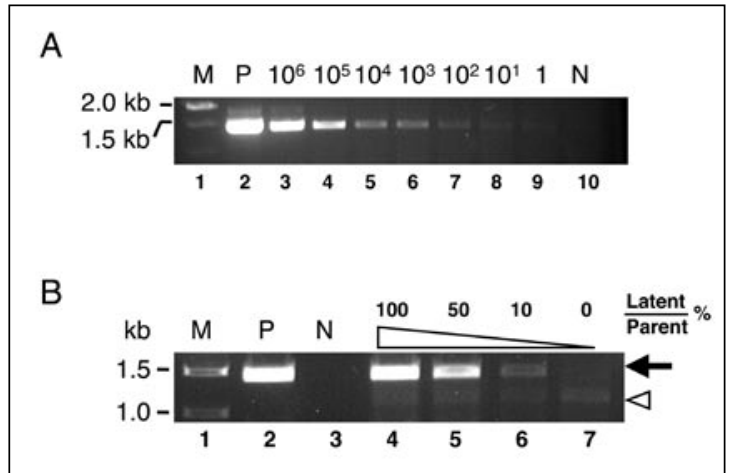

Figure 2. Assay sensitivity. (A) Detection of adeno-associated virus (AAV) viral DNA contamination in cell lysates. Cell lysates $\left(2 \times 10^{6}\right.$ cells $/ 100 \mu \mathrm{L}$ TD buffer) from AAV-free cells were spiked with 10 -fold serial dilutions of purified wild-type AAV ranging from $10^{8}$ to 100 particles. After DNA extraction and elution in $100 \mu \mathrm{L}$ of water, $1 \mu \mathrm{L}$ of the elution was used in a PCR of which one-tenth was run on a $0.7 \%$ agarose gel. M, molecular size marker; P, AAV2 helper plasmid; $10^{6}$ and 1, copy number of the wild-type AAV2 in $1 \mu \mathrm{L}$ of the original lysates; $\mathrm{N}$, no template DNA (the PCR mixture only). (B) Detection of AAV contamination in latently infected tissue culture cells. Genomic DNA was isolated from either AAV2 latently infected cells or the parental cells $\left(2 \times 10^{6}\right.$ cells $)$ and used as input material for PCR with the AAV-specific primers. Twenty microliters of each PCR product was applied on the gel. The position of the specific PCR product is indicated by an arrow. A lower molecular weight nonspecific genomic DNA PCR band is indicated by an open triangle. M, molecular size marker; P, AAV2 helper plasmid; N, no template DNA control.

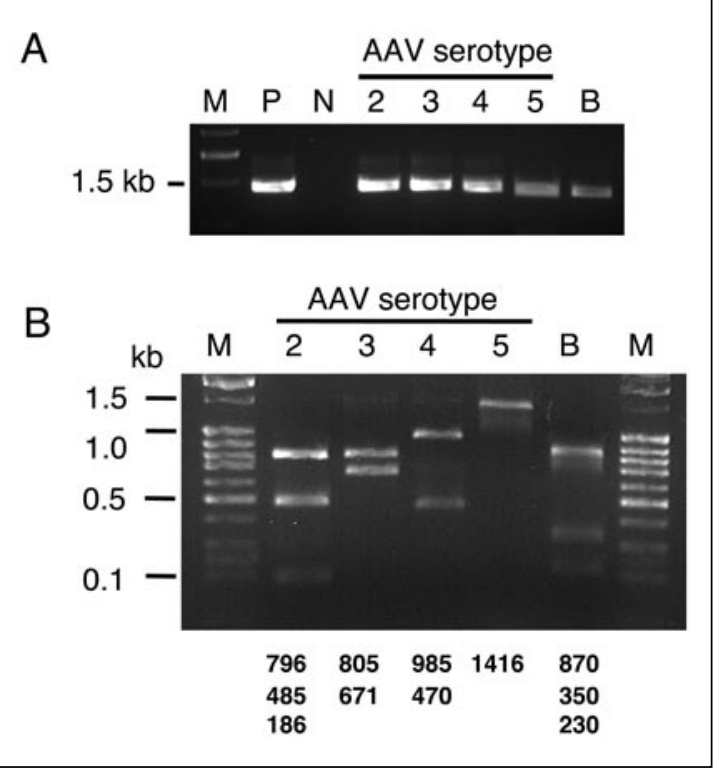

Figure 3. Detection of a new isolate of adeno-associated virus (AAV) from cleared viral lysates (CVLs). (A) Bovine adenovirus (Ad) stocks were obtained from the ATCC (accession no. VR-314). A 1.4-kb band was detected after adding $1 \mu \mathrm{L}$ of the viral stock to the PCR assay. M, molecular size marker; P, purified wild-type AAV2; N, no DNA; 2, recombinant AAV2-CVL (rAAV2- CVL); 3, rAAV3- CVL; 4, rAAV4- CVL; 5, rAAV5CVL; B, bovine Ad stock. (B) Discrimination between serotypes. Following PCR, the mixture was treated with $\mathrm{XhoI}$ and analyzed by $2 \%$ agarose gel electrophoresis. The sizes of the fragment(s) in base pairs are indicated on the bottom. find significant homology with other autonomous parvoviruses such as B19 (data not shown). The total PCR product spans nucleotides 1437 to 2904 in the AAV2 genome and produces a 1.4$\mathrm{kb}$ product in AAVs $1-6$ and 8. A $1.5-$ or $1.6-\mathrm{kb}$ product is produced with AAV7 or AAAV, respectively.

Initial experiments with this primer set produced products of the expected size when tested with CVLs prepared in the laboratory containing either AAV2-6 or AAAV. An improvement in amplification sensitivity and specificity was observed if the DNA was first purified from the samples using the QIAprep Spin Miniprep kit with the following modification. The volume of P1 buffer was reduced to account for the $100-\mu \mathrm{L}$ volume of the CVL (Figure 1C, lane 4-8). To determine the sensitivity of the assay, samples of cleared cell lysate from virus-free 293T cells were spiked with aliquots of purified wild-type AAV2. Prior to PCR, the viral DNA was purified from the spiked cell lysate $\left(2 \times 10^{6}\right.$ cells $/ 100 \mu \mathrm{L}$ ) as described above. A specific AAV band could easily be detected in samples containing $10^{4}$ viral genomes (Figure 2A, lane 5) using the universal primer set with sensitivity down to 10 particles/reaction or 10 particles $/ 2 \times 10^{4}$ cells (Figure 2A, lane 8). The sensitivity of this PCR assay was also tested with cleared cell lysate spiked with either AAV4 or AAV5. AAV4 differs from AAV2 only in primer 2 , while AAV5 differs from AAV2 in both primer 1 and primer 2. In these experiments, a specific AAV4 band could be detected at 100 copies/ reaction, while AAV5 was sensitive to only 1000 copies/reaction (data not shown). The AAV5 data is in agreement with the difference in product band intensity for AAV2 and AAV5 in Figure 1C.

In the absence of a helper virus, AAVs are able to establish latency in the infected cell. To test the utility of the AAV-specific primer set for detection of AAV contamination in latently infected tissue culture cells, $4 \times 10^{5}$ cells were infected with purified AAV2 at an MOI of 5000. Forty-eight hours postinfection, individual cell clones were isolated by limiting dilution and maintained for 8 weeks (greater than three cell passages) prior to assaying for AAV DNA. Latently infected clones were identified by their ability to produce wild-type AAV particles upon super infection with Ad. Total genomic DNA $\left(2 \times 10^{6}\right.$ cells $)$ was extracted using the QIAamp ${ }^{\circledR}$ Blood DNA Mini kit (Qiagen) as described by the manufacturer. A robust PCR band was observed following the addition of 5 cycles to the second round of PCR ( 30 cycles instead of 25). The specific AAV product could be detected in PCRs containing $1 \mu \mathrm{L}$ of the input DNA corresponding to $2 \times 10^{4}$ cells (Figure 2B, lane 4). To determine the sensitivity of this assay, purified DNA from the latently infected cells was mixed with DNA from the parent cells at different ratios and amplified by PCR. Using this method, AAV contamination could still be detected in samples of latent infected cells that were diluted 1:10 with DNA isolated from uninfected cells (Figure 2B, lane 6). Thus, AAV contamination in either the virus stocks, the cleared cell lysate, or the latently infected cells can be detected using these universal primers.

Of the eight cloned AAV isolates, five were originally identified as contaminants of laboratory stocks of Ad. To screen for AAV contamination in other laboratory stocks of Ad, stocks of Ad CVL (VR314) were obtained from ATCC (Manassas, VA, USA) that were reported to be contaminated with AAV.

Following PCR amplification, a characteristic $1.4-\mathrm{kb}$ band was detected with the DNA isolated from the bovine Ad CVL (Figure 3A, lane 8). However, digestion of PCR products with the restriction enzyme XhoI gave a unique banding pattern compared to that of 
other known AAV isolates, suggesting that this Ad stock contained a unique AAV isolate (Figure 3B, lane 6).

\section{DISCUSSION}

AAV contamination in laboratory stocks of virus is known to effect their growth and metabolic activity (7). Therefore, the identification of AAVfree material is an important experimental consideration. In this article, we describe a PCR-based method that is both sensitive and specific for a broad array of AAV isolates. With this AAV-specific primer set and optimized DNA preparation, five molecules of AAV DNA $/ 10^{4}$ cells can be detected in a sample of virus stocks or tissue. Some variation in sensitivity was noted with different templates, which could be the result of different annealing or extension efficiency of the primers. The establishment of an assay for the detection and cloning of AAV from viral and cellular samples will help to facilitate the understanding of the biology of the dependent parvovirus family as well as identify contamination in laboratory samples.

\section{ACKNOWLEDGMENTS}

The authors wish to acknowledge the contributions of Beverly Handelman for technical assistance and Roberta Knox and Karen Knight for administrative assistance. We thank Giovanni Dipasquale and Roland Owens for critical review and helpful discussions of this manuscript.

\section{REFERENCES}

1.Muzyczka, N. and K.I. Berns. 2001. Fields Virology. Lippincott, Williams \& Wilkins, Philadelphia.

2.Carter, B.J., C.A. Laughlin, L.M. de la Maza, and M. Myers. 1979. Adeno-associated virus autointerference. Virology 92:449462.

3.Casto, B.C., J.A. Armstrong, R.W. Atchison, and W.M. Hammon. 1967. Studies on the relationship between adeno-associated virus type 1 (AAV-1) and adenoviruses. II. Inhibition of adenovirus plaques by AAV; its nature and specificity. Virology 33:452-458.

4.Casto, B.C., R.W. Atchison, and W.M. Hammon. 1967. Studies on the relationship between adeno-associated virus type I (AAV-
1) and adenoviruses. I. Replication of AAV-1 in certain cell cultures and its effect on helper adenovirus. Virology 32:52-59.

5.Weitzman, M.D., K.J. Fisher, and J.M. Wilson. 1996. Recruitment of wild-type and recombinant adeno-associated virus into adenovirus replication centers. J. Virol. 70:18451854.

6.Yang, Q., F. Chen, J. Ross, and J.P. Trempe. 1995. Inhibition of cellular and SV40 DNA replication by the adeno-associated virus Rep proteins. Virology 207:246-250.

7.Di Pasquale, G. and J.A. Chiorini. 2003. PKA/PrKX activity is a modulator of AAV/ adenovirus interaction. EMBO J. 22:17161724.

8.Bantel-Schaal, U. and H. zur Hausen. 1988. Adeno-associated viruses inhibit SV40 DNA amplification and replication of herpes simplex virus in SV40-transformed hamster cells. Virology 164:64-74.

9.Hermonat, P.L. 1992. Inhibition of bovine papillomavirus plasmid DNA replication by adeno-associated virus. Virology 189:329333.

10.Hermonat, P.L. 1994. Adeno-associated virus inhibits human papillomavirus type 16 : a viral interaction implicated in cervical cancer. Cancer Res. 54:2278-2281.

11.Antoni, B.A., A.B. Rabson, I.L. Miller, J.P. Trempe, N. Chejanovsky, and B.J. Carter. 1991. Adeno-associated virus Rep protein inhibits human immunodeficiency virus type 1 production in human cells. J. Virol. 65:396404.

12.Rittner, K., R. Heilbronn, J.A. Kleinschmidt, and G. Sczakiel. 1992. Adeno-associated virus type 2-mediated inhibition of human immunodeficiency virus type 1 (HIV1) replication: involvement of p78rep/p68rep and the HIV-1 long terminal repeat. J. Gen. Virol. 73:2977-2981.

13.Hermonat, P.L. 1989. The adeno-associated virus Rep78 gene inhibits cellular transformation induced by bovine papillomavirus. Virology 172:253-261.

14.Khleif, S.N., T. Myers, B.J. Carter, and J.P. Trempe. 1991. Inhibition of cellular transformation by the adeno-associated virus rep gene. Virology 181:738-741.

15.Labow, M.A. and K.I. Berns. 1988. The adeno-associated virus rep gene inhibits replication of an adeno-associated virus/simian virus 40 hybrid genome in cos-7 cells. J. Virol. 62:1705-1712.

16.Kaludov, N., K.E. Brown, R.W. Walters, J. Zabner, and J.A. Chiorini. 2001. Adeno-associated virus serotype 4 (AAV4) and AAV5 both require sialic acid binding for hemagglutination and efficient transduction but differ in sialic acid linkage specificity. J. Virol. 75:6884-6893.

17.Alisky, J.M., S.M. Hughes, S.L. Sauter, D. Jolly, T.W. Dubensky, Jr., P.D. Staber, J.A. Chiorini, and B.L. Davidson. 2000. Transduction of murine cerebellar neurons with recombinant FIV and AAV5 vectors. Neuroreport 11:2669-2673.

18.Fisher, K.J., K. Jooss, J. Alston, Y. Yang, S.E. Haecker, K. High, R. Pathak, S.E. Raper, and J.M. Wilson. 1997. Recombinant adeno-associated virus for muscle directed gene therapy. Nat. Med. 3:306-312.

19.Flotte, T.R. 1993. Prospects for virus-based gene therapy for cystic fibrosis. J. Bioenerg. Biomembr. 25:37-42.

20.Snyder, R.O., S.K. Spratt, C. Lagarde, D. Bohl, B. Kaspar, B. Sloan, L.K. Cohen, and O. Danos. 1997. Efficient and stable adenoassociated virus-mediated transduction in the skeletal muscle of adult immunocompetent mice. Hum. Gene Ther. 8:1891-1900.

21.Gao, G., M.R. Alvira, S. Somanathan, Y. Lu, L.H. Vandenberghe, J.J. Rux, R. Calcedo, J. Sanmiguel, et al. 2003. Adeno-associated viruses undergo substantial evolution in primates during natural infections. Proc. Natl. Acad. Sci. USA 100:6081-6086.

22.Jeanmougin, F., J.D. Thompson, M. Gouy, D.G. Higgins, and T.J. Gibson. 1998. Multiple sequence alignment with Clustal $\mathrm{X}$. Trends Biochem. Sci. 23:403-405.

23.Chiorini, J.A., F. Kim, L. Yang, and R.M. Kotin. 1999. Cloning and characterization of adeno-associated virus type 5. J. Virol. 73:1309-1319.

Received 7 October 2003; accepted 20 January 2004.

Address correspondence to John A. Chiorini, NIH 10/1N113, 10 Center Dr., MSC1190, Bethesda, MD 20892, USA. e-mail: jchiorini@dir.nidcr.nih.gov 